\title{
Les systèmes herbagers économes. Une alternative de développement agricole pour l'élevage bovin laitier dans le Bocage vendéen?
}

Grassland inputs-saving systems: an alternative for agricultural development of dairy cattle farming in Bocage of Vendée?

\section{Nadège Garambois et Sophie Devienne}

\section{OpenEdition}

\section{Journals}

Édition électronique

URL : http://journals.openedition.org/economierurale/3496

DOI : 10.4000/economierurale.3496

ISSN : 2105-2581

\section{Éditeur}

Société Française d'Économie Rurale (SFER)

Édition imprimée

Date de publication : 30 juillet 2012

Pagination : $56-72$

ISSN : 0013-0559

Référence électronique

Nadège Garambois et Sophie Devienne, «Les systèmes herbagers économes. Une alternative de développement agricole pour l'élevage bovin laitier dans le Bocage vendéen ? », Économie rurale [En ligne], 330-331 | juillet-septembre 2012, mis en ligne le 30 juillet 2014, consulté le 19 avril 2019. URL http://journals.openedition.org/economierurale/3496; DOI : 10.4000/economierurale.3496 


\section{Les systèmes herbagers économes Une alternative de développement agricole pour l'élevage bovin laitier dans le Bocage vendéen ?}

Nadège GARAMBOIS, Sophie DEVIENNE • UFR Agriculture comparée et développement agricole, AgroParisTech, Paris

nadege.garambois@agroparistech.fr ; sophie.devienne@agroparistech.fr

D epuis les années 1950, le développement agricole de l'Ouest de la France a été marqué par la spécialisation des exploitations dans l'élevage, la concentration de la production dans un nombre toujours plus restreint d'exploitations de plus en plus grandes, l'accroissement de la production et une forte progression de la productivité physique du travail. Celle-ci a reposé sur l'agrandissement de la superficie des exploitations et de la taille des troupeaux, mais aussi sur l'accroissement de la production par hectare, permis par le recours croissant aux consommations intermédiaires et par l'évolution des systèmes fourragers vers une utilisation grandissante du maïs fourrage et une réduction de la place des prairies et du pâturage.

En rupture avec ce développement, certains éleveurs ont mis en place dans l'Ouest français des systèmes bovins laitiers qualifiés d' « herbagers » car majoritairement basés sur l'utilisation des prairies. Motivés par la volonté d'accroître l'autonomie et la durabilité de leur exploitation, ces éleveurs ont modifié en profondeur leurs systèmes en développant les prairies temporaires (association graminées-légumineuses) et la place du pâturage dans l'alimentation, tout en réduisant le recours au maïs, à la fertilisation azotée et à la complémentation en tourteau. Ce mouvement, qui concerne aujourd'hui en France près de 2000 éleveurs, a démarré dans les Côtes d'Armor, impulsé par l'agriculteur André Pochon (Pochon, 2002). Différentes études technico-économiques conduites à l'échelle départementale ou régionale (Alard,
Béranger, Journet, 2002 ; Le Rohellec, Mouchet, 2004 ; Le Rohellec, 2008) basées sur la comparaison de moyennes entre un « groupe herbager» et un groupe témoin (échantillon « Rica-Otex bovin lait» ou exploitations adhérentes à un centre de gestion local) montrent que ces systèmes herbagers permettent d'obtenir un revenu moyen satisfaisant.

L'objectif de cette recherche (Garambois, 2011) était notamment d'affiner l'analyse de ces transformations, en replaçant les systèmes herbagers dans le temps long du développement agricole, afin d'en comprendre l'émergence, d'identifier, dans leur diversité, les différents systèmes bovins laitiers actuels, qu'ils soient « herbagers » ou non, et d'en comparer le fonctionnement et les résultats économiques. Ces travaux se proposent, grâce à l'analyse des performances économiques des systèmes de production en relation étroite avec leur fonctionnement technique, de comprendre la logique spécifique de fonctionnement des systèmes herbagers économes et de mettre en lumière la forme particulière d'intensification développée par ces éleveurs. La dimension diachronique de l'analyse conduit également à s'interroger sur les perspectives d'évolution des différents systèmes bovins et sur la pertinence de cette voie radicalement différente de développement agricole.

Afin de comparer des systèmes de production mis en œuvre dans des conditions agro-écologiques et socio-économiques homogènes, l'échelle de la petite région agricole a été retenue. Le choix a porté sur le Bocage vendéen, petite région 
du Centre Ouest où une centaine d'éleveurs herbagers organisés en plusieurs groupes locaux ${ }^{1}$ ont élaboré à partir de 1990 des systèmes basés sur des prairies temporaires de graminées et légumineuses, dans des conditions climatiques moins favorables à la pousse de l'herbe que celles des Côtes d'Armor, en raison d'un déficit hydrique souvent important en été.

\section{Matériel et méthode}

Cette recherche repose sur la méthode d'analyse-diagnostic des systèmes agraires développée par l'UFR d'Agriculture Comparée et Développement agricole (Mazoyer, Roudart, 1997 ; Dufumier, 1996 ; Cochet, Devienne, 2006 ; Cochet, Devienne, Dufumier, 2007) et mobilise le concept de système de production (Cochet et al., 2007). Ce concept s'applique ici à un ensemble d'exploitations possédant la même gamme de ressources et pratiquant une combinaison similaire et spécifique de systèmes de culture et de systèmes d'élevage (Reboul, 1976). Un système de production est une «représentation finalisée du réel » (Landais, 1992), une modélisation de la réalité dont l'objectif est de tenter de comprendre le fonctionnement, l'origine et les perspectives d'évolution d'un type d'exploitation donné. Cette représentation ne s'appuie pas sur une « moyenne » mais sur la compréhension d'une logique de fonctionnement et d'organisation de la production. La modélisation $\mathrm{du}$ fonctionnement technique du système de production est la base sur laquelle peuvent être reconstruits, dans un second temps, ses résultats économiques modélisés (Cochet et al., 2006) (cf. annexe).

1. GRADEL (Groupe de Recherche en Agriculture Durable et en Économie Locale), Civam HB (Centre d'Initiatives pour Valoriser l'Agriculture et le Milieu rural du Haut-Bocage), GRAPEA (Groupe de Recherche pour une Agriculture Paysanne Économe et Autonome).
L'étude a été conduite sur la base d'une analyse-diagnostic (Cochet et al., 2006 ; Cochet et al., 2007) de l'agriculture de la petite région du Bocage vendéen, grâce à un travail de terrain approfondi mené entre mars 2007 et avril 2009. La reconstitution de la dynamique historique de l'agriculture de la région a reposé sur une trentaine d'entretiens auprès d'agriculteurs ayant été les acteurs et témoins de ces transformations, complétés des statistiques disponibles. La compréhension des relations entre différenciation sociale et changements techniques permet de comprendre le processus de différenciation des systèmes de production (Dufumier, Bergeret, 2002), de reconstruire leurs trajectoires d'évolution, d'identifier sur cette base les différents systèmes de production actuels et d'en bâtir une typologie. Cette typologie, qui est ensuite progressivement affinée, constitue la base de départ pour l'élaboration d'un échantillon raisonné des exploitations à enquêter, permettant de rendre compte de l'ensemble des systèmes de production identifiés. Les enquêtes conduites visent à la fois à caractériser le fonctionnement technico-économique des systèmes de production et à comprendre les causes et modalités de leur évolution afin de compléter et d'affiner la typologie établie. Cette démarche itérative a pour objectif de repérer et de comprendre la diversité des systèmes de production de la région et d'expliciter, modéliser et comparer la logique de fonctionnement et les résultats techniques et économiques de chacun d'entre eux.

Sur la base de cet échantillonnage raisonné, une centaine d'enquêtes approfondies ont été effectuées auprès d'exploitations agricoles du Bocage vendéen spécialisées en élevage bovin, dont le tiers avait évolué vers un système herbager. Les résultats présentés ici concernent le seul élevage bovin laitier au travers d'une cinquantaine d'enquêtes d'exploitations majoritairement situées dans le canton de Rocheservière. 


\section{Le contexte d'émergence de systèmes alternatifs}

\section{Priorité à l'accroissement de la productivité physique du travail}

Le développement de la production laitière dans la région depuis les années 1950 s'est caractérisé par une forte augmentation de la production de lait par actif, reposant essentiellement sur la spécialisation, l'accroissement de la capacité des équipements et le recul du pâturage au profit du maïs fourrage. Durant cette période, la baisse des prix réels des produits agricoles a été importante et plus rapide que celle des biens nécessaires au processus de production agricole (Boucarut, Moyne, Pollina, 1996 ; Guihard, Lesdos, 2007). Les exploitations qui avaient les moyens de réaliser des investissements productifs ont cherché à compenser les effets négatifs de la baisse des prix agricoles sur leur revenu en accroissant leur production et la productivité physique de leur travail (Desriers, 2007), aidée dans ce mouvement par la sécurité des prix offerte dans certains secteurs de production par la Politique Agricole Commune.

Dès l'après-guerre, les agriculteurs du Bocage vendéen, qui mettaient en œuvre des systèmes de polyculture-élevage déjà fortement orientés vers l'élevage bovin, ont cherché à augmenter la production par hectare et par actif, en s'agrandissant et en transformant leur système d'élevage. L'acquisition d'équipements plus performants, tant pour les cultures que pour l'alimentation et la traite des animaux, ainsi que la modernisation et l'accroissement de la capacité des bâtiments ont parallèlement rendu possible l'augmentation de la superficie des exploitations et du nombre de vaches par actif. Cette substitution continue du capital au travail s'est accompagnée de l'éclatement du système de polyculture-élevage en systèmes de production spécialisés, processus de spécialisation qui, en retour, a contribué également à l'accroissement du nombre de vaches par actif.
L'adoption du tracteur, le développement de l'utilisation des amendements et engrais dès les années 1950, puis l'arrachage des haies et le regroupement du parcellaire au cours des années 1960, qui ont été permis par le remembrement et ont facilité la progression de la motorisation des opérations culturales, ont ouvert la voie à de profondes transformations : les agriculteurs ont substitué aux prairies permanentes et aux plantes sarclées fourragères exigeantes en main-d'œuvre, des prairies temporaires de graminées plus productives (ray-grass anglais dès les années 1950 et ray-grass d'Italie (RGI) à partir des années 1960) et, partant, augmenté la taille de leur troupeau et remplacé les vaches Charolaises par des races à plus haut potentiel laitier (Normande, puis Pie noire).

Avec l'introduction dans la région des premières variétés de maïs hybride dès le milieu des années 1960, les gains de productivité du travail ont ensuite reposé sur le recul progressif du pâturage au profit de l'accroissement de la part du maïs fourrage dans l'alimentation. Cette culture s'est développée rapidement, au sein de rotations de type maïs/blé/RGI 6 ou 18 mois, pour atteindre près du quart de la Surface Agricole Utile (SAU) à la fin des années $1970^{2}$. Elle présente en effet l'avantage d'offrir un rendement et une densité énergétique plus élevés, d'être entièrement moto-mécanisable et de pouvoir être stockée sous forme d'ensilage, augmentant ainsi les disponibilités fourragères toute l'année, accompagnant ainsi efficacement la progression du potentiel de rendement laitier des troupeaux (changement de race, sélection génétique). L'accroissement du chargement permis par l'augmentation de la production fourragère par hectare, qu'accompagnait éventuellement l'agrandissement des exploitations, a conduit les éleveurs qui en avaient les moyens à développer de manière impor-

2 à 7. Données Recensement Général Agricole (RGA), canton de Rocheservière (Bocage vendéen). 
tante la capacité de leurs équipements (salle de traite de $2 \times 3$ à $2 \times 6$ postes) et à moderniser leurs bâtiments (stabulation libre avec couloir central d'alimentation) afin de faciliter la moto-mécanisation de la distribution des fourrages et du curage.

En drainant une grande partie de leurs terres durant les années 1980 et $1990(1 \%$ de la SAU en 1979, $33 \%$ en 1988 et $56 \%$ en $2000^{3}$ ), les agriculteurs ont stabilisé le rendement du maïs fourrage et converti en prairies temporaires, voire mis en culture, l'essentiel des prairies permanentes restantes, qui ne représentaient en moyenne plus que $10 \%$ de la SAU en $2000^{4}$. L'investissement dans l'irrigation grâce à des retenues partiellement alimentées par les eaux de drainage ( $1 \%$ de la SAU était irrigable en 1970 et $14 \%$ en $2000^{5}$ ) a permis quant à lui de faire encore progresser les rendements du maïs fourrage. La progression continue de la part de l'ensilage de maïs et des compléments en céréales et tourteau de soja dans l'alimentation du troupeau, alliée à l'adoption de la race Holstein, a rendu possible la poursuite de l'accroissement des rendements laitiers.

Le contingentement de la production laitière à partir de 1984 n'a pas enrayé ce mouvement d'accroissement de la production par hectare prévalant depuis l'aprèsguerre. La progression continue des rendements et de la densité énergétique de l'alimentation a permis aux éleveurs de réaliser leur production laitière en réduisant leur cheptel (baisse de $10 \%$ des effectifs de vaches laitières entre 1979 et 1988 et de $21 \%$ entre 1988 et $2000^{6}$ ) ainsi que la superficie dédiée à cette production. Les surfaces ainsi libérées ont été valorisées par d'autres productions : les céréales, dont les surfaces sont passées de $20 \%$ de la SAU en 1970 et 1988 à $27 \%$ en $2000^{7}$, ou l'engraissement des veaux mâles en taurillons nourris à l'ensilage de maïs, lorsque les éleveurs disposaient d'une plus faible surface par actif. L'octroi de primes spécifiques à ces productions dans le cadre de la réforme de la PAC de 1992 a contribué à renforcer ces orientations.

Les exploitations de polyculture-polyélevage à traction attelée du Bocage vendéen des années 1950 (Dumont, Coléou, Séverac, 1957) ont ainsi connu, en soixante ans, un accroissement spectaculaire des rendements des cultures comme de la production laitière par vache (jusqu'au quintuple), tandis que dans le même temps la surface cultivée par actif augmentait au moins d'un facteur six, multipliant par trente la productivité physique du travail entre 1950 à 2010.

\section{Des éleveurs à la recherche d'une plus grande autonomie}

Le développement agricole régional, centré sur l'accroissement de la productivité physique du travail, a conduit à une concentration de la production dans un nombre toujours plus réduit d'exploitations dont la taille n'a cessé de croître. Ces agrandissements ont été permis par la disparition progressive et continue des exploitations qui n'ont pas eu les moyens de suivre ce mouvement, faute d'une superficie suffisante dans les années 1950, d'opportunités d'agrandissement par la suite et de la capacité d'investissement nécessaire (Mazoyer, 1981 ; Mounier, 1992). Le nombre d'exploitations dans le canton de Rocheservière a ainsi diminué de $67 \%$ entre 1970 et 2000 et cette baisse est encore plus marquée $(-76 \%)$ dans le cas des exploitations spécialisées en élevage bovin laitier ${ }^{8}$. Au plan économique, ce développement agricole repose sur une externalisation accrue des tâches agricoles et sur un recours toujours plus important aux intrants et à des équipements de plus en plus performants. La production importante par unité de surface est permise par des consommations intermédiaires et de capital fixe élevées, qui représentent désormais respectivement de 55 à $65 \%$ et de 15 à $25 \%$ du produit brut des différents systèmes de

8. Recensement Général Agricole. 
production laitiers de la région au sein desquels la conduite du troupeau au pâturage n'excède pas trois mois.

Certains agriculteurs du Bocage vendéen ont constaté, dès la fin des années 1980, que les consommations intermédiaires et les immobilisations de capital fixe grevaient de plus en plus lourdement leur revenu et se sont inquiétés des impacts environnementaux liés à l'usage, qu'ils jugeaient excessif, d'engrais azoté et de pesticides. Ils ont cherché à mettre en œuvre des systèmes plus autonomes et économes en intrants, qui leur permettraient de maintenir leur revenu en réduisant leurs coûts plutôt qu'en augmentant le produit brut par actif, et d'alléger en outre leur charge en travail. Ils se sont appuyés dès le début des années 1990 sur le référentiel technique des systèmes herbagers bretons (Pochon, 2002), mis au point dans des conditions particulièrement favorables à la pousse de l'herbe, qu'il leur a fallu adapter aux conditions édaphiques et climatiques du Bocage vendéen, pour la conduite du système d'élevage (période de vêlage, systèmes fourragers) comme des systèmes de culture (choix des espèces et variétés de graminées et de légumineuses associées, conduite des prairies). À l'instar de la Bretagne, la constitution de groupes locaux a largement favorisé les échanges entre agriculteurs (Deléage, 2004) et joué un rôle déterminant dans l'optimisation des techniques et des savoir-faire.

\section{Le développement à contre-courant des systèmes herbagers économes}

\section{Une logique de fonctionnement \\ très différente}

À l'issue de soixante années d'évolution et de différenciation des systèmes de production, les systèmes spécialisés en élevage bovin laitier prédominant aujourd'hui dans le Bocage vendéen se caractérisent par la place réduite du pâturage et par un haut niveau de performance technique. Les systèmes de culture et d'élevage présentent de grandes similitudes : mêmes types de rotations (maïs/blé/RGI 18 mois et maïs/blé) conduites sur plus de $65 \%$ de la superficie des exploitations, les $35 \%$ restants étant occupés par des prairies temporaires (ou permanentes) ; itinéraires techniques des cultures semblables; rôle central de l'ensilage de maïs dans les systèmes fourragers au détriment du pâturage ; rendements laitiers supérieurs à 8000 litres ; part importante de la superficie (20\% à $55 \%)$ consacrée à des céréales destinées à la vente.

À rebours du mouvement de réduction de la pâture et de la mobilité des troupeaux observés dans la région, les systèmes herbagers économes ont une logique de fonctionnement très différente. Ils placent au cœur de leur fonctionnement les prairies temporaires associant graminées et trèfle blanc au rendement élevé et conduites sans azote de synthèse, ainsi que le pâturage, pratiqué suivant les techniques de pâturage tournant (Civam, 2009). Le troupeau pâture neuf mois par an, la moitié du temps sans complément fourrager, et la ration hivernale ne comprend qu'un tiers d'ensilage de maïs. Malgré le pic de croissance de l'herbe au printemps, la période de vêlages a été maintenue à l'automne afin de tarir le troupeau en été, période d'étiage fourrager des prairies nettement plus marqué dans le Bocage vendéen qu'en Bretagne, et de bénéficier d'un prix moyen du lait semblable à celui des systèmes de production reposant moins sur le pâturage. Les éleveurs herbagers ont conservé la race Holstein car la plasticité de sa production lui permet de réaliser un rebond de la courbe de lactation avec la mise au pâturage au début du printemps, et de profiter ainsi, en fin de lactation, de la pousse rapide de l'herbe à cette saison, tout en maintenant un rendement laitier annuel relativement élevé, variant, suivant le niveau de complémentation, de 6000 à 7500 litres par vache et une production de lait de 4000 à 5500 litres par hectare de SAU. 
En rupture avec le développement agricole prédominant dans la région, ces éleveurs ont fait le choix de réaliser leurs quotas laitiers avec un cheptel plus important, en lui consacrant l'ensemble de la surface agricole, sans produire de cultures de vente. Les surfaces en maïs et blé ont ainsi été réduites au profit des prairies temporaires d'association et la rotation maïs/blé/RGI 18 mois remplacée par une rotation longue maïs/triticale ou mélange céréalier/prairies temporaires graminéeslégumineuses de six à dix ans. La ration, moins dense sur le plan énergétique, n'autorise qu'un rendement laitier par vache plus faible, mais elle présente l'avantage d'être bien moins coûteuse. L'herbe des prairies temporaires de longue durée à base de légumineuses constitue en effet plus de $80 \%$ des fourrages produits (deux fois plus que dans les systèmes avec pâturage au printemps de prairies de graminée pure), et elle est prélevée pour les trois quarts directement par l'animal au pâturage. Ces fourrages plus équilibrés en protéines et le plus faible objectif de rendement laitier permettent par ailleurs de réduire fortement les achats de tourteau.

\section{Une différenciation structurelle bien moins marquée}

L'homogénéité des systèmes de culture et d'élevage caractérisant les systèmes de production mis en œuvre au sein de la plupart des exploitations spécialisées en élevage bovin laitier du Bocage vendéen contraste avec leur profonde différenciation structurelle. Les différences d'accès au foncier (notamment la superficie par actif) ainsi qu'aux références laitières (quotas par hectare) ont en effet conduit à une différenciation marquée des systèmes de production.

Certains éleveurs ont réussi à compenser la faible surface dont ils disposaient (moins de 35 ha par actif jusqu'à la fin des années 1990) en augmentant la production de lait par hectare, notamment grâce à des inves- tissements précoces dans l'irrigation. Les références laitières élevées par unité de surface dont ils ont disposé lors de la mise en place des quotas en 1984 ont été accrues depuis, grâce à un agrandissement modeste et à une reprise de quotas importante lors du renouvellement des actifs, et sont désormais comprises entre 6000 et 7000 litres par ha de SAU. Ils parviennent à réaliser ce haut niveau de production en adoptant une alimentation en zéro pâturage. Celle-ci est pratiquée dans des exploitations disposant d'une superficie de 60 à 90 ha pour deux actifs, équipées de $2 \times 6$ postes de traite. Parmi celles-ci, les éleveurs disposant de moins de 35 ha par actif et irrigant l'ensemble de la surface en maïs mettent en œuvre un système qui permet d'atteindre 9500 à 10000 litres de lait produit par vache grâce à une ration fourragère reposant intégralement sur l'ensilage de maïs (système de production (SP) 1). Avec 35 à 45 ha par actif, mais sans accès à l'irrigation, les éleveurs ont maintenu $20 \%$ d'ensilage d'herbe dans l'alimentation du troupeau, composée sinon de maïs fourrage, et atteignent un rendement laitier de 9000 à 9500 litres (SP2).

La plupart des éleveurs de la région ont pu agrandir davantage leur exploitation et accéder à une plus grande superficie par actif, passant de 35 à 45 ha dans les années 1990 à 40 à 60 ha aujourd'hui et disposent de références laitières par hectare de SAU plus modestes, comprises entre 4000 et 5000 litres. De nombreux éleveurs ont recours à l'irrigation, mais les équipements et parfois la dispersion du foncier liée aux agrandissements successifs, ne permettent pas d'irriguer plus de $50 \%$ de la surface en maïs. Ils continuent à pratiquer un pâturage de printemps, période de l'année où la pousse de l'herbe est la plus rapide et où les besoins du troupeau sont moindres, puisque les vaches sont en fin de lactation (vêlages d'automne). L'essentiel de la production laitière est réalisée en automne et en hiver, grâce à une alimentation reposant sur trois 
quarts d'ensilage de maïs et un quart d'ensilage d'herbe, largement complétés de tourteaux de soja et de céréales, qui permet d'atteindre des rendements laitiers de 8500 à 9000 litres. Les inégalités d'accès aux ressources, notamment au foncier, ont cependant présidé à des trajectoires d'exploitations distinctes et conduit à une différenciation des systèmes de production reposant sur des niveaux d'équipement ainsi qu'une gamme de taille de troupeau et de superficie différents :

- système mis en œuvre par des exploitations à un actif disposant de 50 à 60 ha équipées d'une salle de traite $2 \times 4$ postes. Ces exploitations sont les héritières des plus petites unités de production de la région des années 1950 (SP3) ;

- systèmes développés par des exploitations de 80 à 100 ha à deux actifs, équipées de $2 \times 5$ postes de traite, où les veaux mâles sont engraissés afin d'accroître la production et le revenu par hectare (SP4) ;

- systèmes mis en œuvre par des exploitations de 100 à 120 ha à deux actifs, équipées de $2 \times 6$ postes de traite et qui vendent leurs veaux mâles à huit jours (SP5) ;

- système développé par des exploitations de 135 à 165 ha occupant trois actifs, équipées d'une salle de traite de $2 \times 8$ postes, où les veaux mâles sont élevés en taurillons (SP6).

Certains éleveurs de la région, qui étaient à la tête des exploitations les plus grandes dans les années 1960, ont eu la possibilité d'accroître encore leur superficie. Disposant dès les années 1990 de 45 à 55 ha par actif, ils pratiquent aujourd'hui un système avec pâturage de printemps au sein d'unités de production dotées de quatre actifs, de 220 à plus de 300 ha répartis en plusieurs îlots et équipées d'au moins 20 postes de traite. Ces exploitations rassemblent des quotas laitiers considérables (de 750000 à plus d'un million de litres) et la taille des troupeaux (90 à 140 vaches) a conduit les éleveurs à automatiser la distribution d'une partie de l'alimentation et à réduire le pâtu- rage au printemps à deux mois, la surface de pâture accessible aux vaches en production étant limitée par le morcellement du parcellaire de ces vastes unités de production. Du fait de leur surface importante (de 55 à 65 ha par actif sur des exploitations de 220 à 260 ha avec une sole de maïs entièrement irriguée (SP7) et jusqu'à 70 à 85 ha par actif (SP8) sur des exploitations de 280 à 340 ha où l'équipement et la répartition spatiale des terres ne permettent pas d'irriguer plus de la moitié de la superficie en maïs), ces éleveurs mettent en œuvre des systèmes à double spécialisation élevage bovin laitier et grandes cultures, au sein desquels ces dernières occupent la moitié des terres.

L'analyse de la différenciation des systèmes de production révèle que, jusqu'en 1990, les éleveurs herbagers avaient connu cette évolution générale et mettaient en œuvre à l'époque les mêmes systèmes de production que les autres éleveurs de la région. Le changement de voie de développement que constitue le passage en système herbager, n'a pas été déterminé par un accès particulier aux facteurs de production. En revanche, l'évolution structurelle qu'ont connue depuis ces exploitations a été très différente puisque leur agrandissement au cours des vingt dernières années a été bien plus modéré. Si ces éleveurs conduisent tous aujourd'hui des systèmes à neuf mois de pâturage, différents systèmes herbagers ont néanmoins émergé, selon la superficie par actif et les quotas laitiers par hectare de SAU dont bénéficiaient les éleveurs avant leur transition.

Certains agriculteurs, installés sur de petites unités de production équipées d'irrigation dès la fin des années 1970, disposaient, avant leur passage en système herbager, de moins de 20 ha par actif et d'importantes références laitières par ha. Ils mettent aujourd'hui en œuvre un système herbager caractérisé par un rendement laitier relativement élevé (7500 litres par vache), grâce au recours resté un peu plus important à l'ensilage de maïs et aux compléments protéiques et céréaliers, au sein d'unités de production de 45 à 60 ha pour un 
et demi à deux actifs, équipées de $2 \times 4$ postes de traite (herb1).

Les autres éleveurs, à la tête d'exploitations plus grandes (20 à 30 ha par actif en 1990) ne se sont jamais équipés d'irrigation et réalisent leurs quotas (plus faibles par unité de surface que dans le système précédent) avec des rendements laitiers un peu moindres, en fournissant moins d'ensilage de maïs, de céréales et de tourteau au troupeau en automne et en hiver. Deux systèmes de production peuvent être distingués parmi ces exploitations. Certains de ces agriculteurs ont maintenu un rendement laitier de 6000 à 6500 litres par vache et disposent de la surface et de la main-d'œuvre suffisantes pour engraisser une partie des veaux en bœufs ou génisses, qui permettent de valoriser les refus du troupeau laitier sur les prairies, tout en nécessitant bien moins de complémentation que les taurillons. Ce système est mis en œuvre au sein d'exploitations qui comptent trois actifs pour 90 à 120 ha, équipées d'une salle de traite $2 \times 6$ postes (herb2). Après le passage à un système herbager, certains de ces éleveurs ont fait le choix de convertir leur exploitation en agriculture biologique dès la fin des années 1990. Le prix du lait supérieur et la recherche d'un rendement laitier relativement important, de l'ordre de 6500 à 7000 litres, ont permis de maintenir le revenu de ces éleveurs avec un agrandissement des exploitations demeuré très modeste (25 à 35 ha par actif) ; ces systèmes de production sont aujourd'hui mis en œuvre au sein d'unités de production qui comptent 60 à 80 ha pour deux à deux actifs et demi et sont équipées de $2 \times 5$ postes de traite (herb3).

Le choix de s'orienter vers un système herbager a également permis à certains éleveurs, au cours des années 2000 , de s'installer de manière individuelle sur des exploitations laitières de petite taille, 40 à 50 ha avec moins de 200000 litres de quotas, qui, sans le passage en système herbager, n'auraient pas pu être reprises et dont la cessation d'activité aurait permis l'agrandisse- ment d'unités de production plus vastes. Ces éleveurs équipés de $2 \times 4$ postes de traite ont conservé ces superficies et quotas peu importants et mettent aujourd'hui en œuvre un système herbager à rendement laitier de 6000 à 6500 litres, sans pratiquer d'engraissement (herb4).

Le développement de systèmes herbagers économes dans le Bocage vendéen a ainsi concerné des exploitations mettant initialement en œuvre des systèmes de production variés, tant sur le plan de la superficie, de la capacité de leurs équipements ou de leurs performances techniques. Le ralentissement de l'agrandissement des unités de production qui a accompagné le passage en système herbager a conduit à une différenciation structurelle moins marquée (exploitations de 40 à 120 ha, équipements de traite de $2 \times 4$ à $2 \times 6$ postes) qui s'exprime par des écarts de superficie par actif limités entre ces différents systèmes.

\section{Des agriculteurs privilégiant la création de valeur ajoutée}

Une valeur ajoutée nette élevée et de moindres subventions

La modélisation des résultats économiques est ici basée sur le système de prix de 2006, retenu plutôt que celui des années 2007 à 2009, marquées par de fortes variations des prix des produits agricoles et des intrants. $\mathrm{La}$ figure 1 compare les résultats économiques des principaux systèmes herbagers à neuf mois de pâturage du Bocage vendéen avec ceux des autres systèmes de production bovins laitiers de la région. Nous avons choisi à cet effet, afin de rendre la comparaison plus aisée, de ne retenir que certains de ces systèmes, qui sont représentatifs des différents grands types de fonctionnement et niveaux de performances techniques atteints : système en zéro pâturage d'une part (SP1) ; systèmes avec pâturage de printemps d'autre part, avec vente des veaux à huit jours (SP5) ou engraissement des veaux mâles en taurillons (SP6) ; système à double 
Figure 1. Résultats économiques à l'hectare des principaux types de systèmes de production bovins laitiers du Bocage vendéen
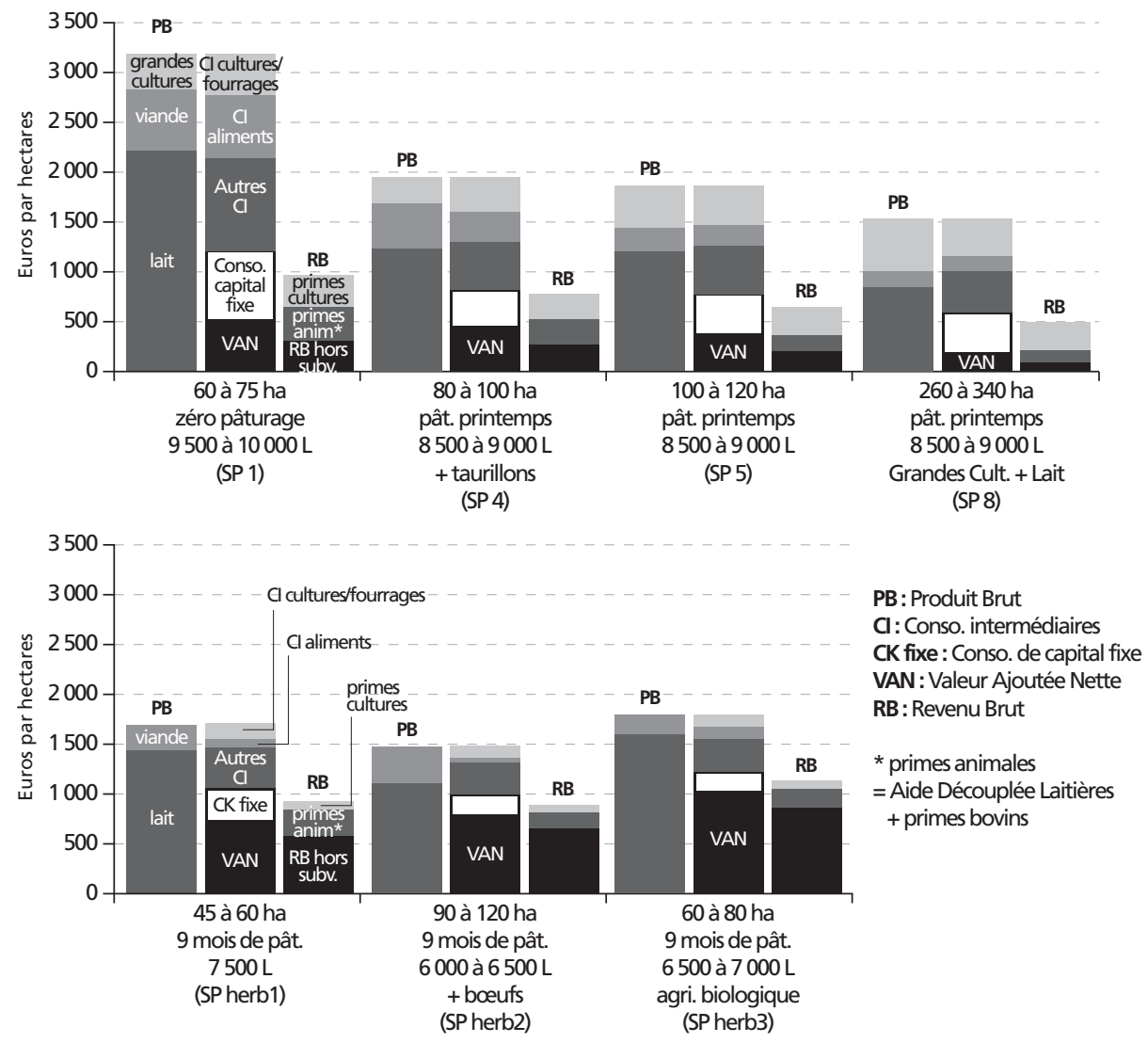

Source : calculs des auteurs

spécialisation élevage bovin laitier et grandes cultures (SP8).

Les systèmes à neuf mois de pâturage dégagent dans leur ensemble un Produit Brut (PB) par hectare inférieur à celui des autres systèmes de production de la région. Ils se caractérisent en revanche par une moindre érosion du PB dans la formation de la Valeur Ajoutée Nette (VAN). La VAN représente ainsi 35 à $55 \%$ du $\mathrm{PB}$ pour ces systèmes herbagers, alors qu'elle ne dépasse pas $25 \%$ du PB pour les autres systèmes de production. Les systèmes herbagers dégagent les VAN par hectare les plus élevées (1,5 à 3 fois celle des autres systèmes laitiers) et pour ces systèmes la VAN contribue à plus de $85 \%$ du
Revenu Brut (RB), contre moins de $65 \%$ pour les autres systèmes et moins de $50 \%$ pour certains d'entre eux (figure 1).

La part plus importante que représente la valeur ajoutée au sein du produit brut s'explique par la forte réduction des coûts de production opérée par les agriculteurs. Ces économies reposent d'une part sur de faibles coûts liés à l'implantation et la conduite des prairies : semences peu onéreuses pour des prairies d'une durée de cinq à dix ans, consommation d'engrais et de pesticides quasi nuls, prélèvement direct de l'herbe par l'animal plutôt que récolte et conservation de fourrages. Les prairies riches en légumineuses offrent de plus une alimentation 
équilibrée aux vaches laitières (Voisin, 1957) et permettent de réduire considérablement le recours aux compléments protéiques achetés. Les consommations intermédiaires ne représentent ainsi que 30 à $40 \%$ du $\mathrm{PB}$, alors qu'elles atteignent 55 à $65 \%$ du $\mathrm{PB}$ pour les autres systèmes de production, tandis que les travaux culturaux moins importants se traduisent par de plus faibles besoins d'équipement et une consommation annuelle moyenne de capital fixe inférieure (figure 1).

Ces systèmes herbagers reçoivent deux fois moins de subventions par hectare, Aide Découplée Laitière (ADL) comprise et hors éventuel Contrat d'Agriculture Durable (CAD) (figure 1). Les autres agriculteurs perçoivent en effet des aides importantes liées aux superficies en maïs fourrage et en cultures de vente, beaucoup plus étendues au sein de leurs systèmes de production. $\mathrm{La}$ part des subventions dans le revenu brut à l'hectare des principaux systèmes de production de la région atteint 55 à $75 \%$, tandis qu'elle n'atteint que 25 à $45 \%$ en système herbager. Le développement de systèmes à double spécialisation bovin laitier et grandes cultures, qui constituent l'étape la plus récente de la progression continue de la productivité physique du travail dans la région, conduit à un renforcement de ces inégalités de soutien, le revenu des agriculteurs mettant en œuvre ces systèmes reposant à hauteur de plus de $85 \%$ sur les subventions.

La valeur ajoutée nette par actif, qui mesure la productivité économique du travail, et le revenu brut par actif peuvent être représentés sur un graphique en fonction de la superficie par actif, permettant ainsi la comparaison des performances économiques des différents systèmes de production. Cette représentation repose sur la modélisation du fonctionnement technique de chaque système de production, à partir de laquelle sont reconstruites, dans un second temps, les performances économiques du système ( $c f$. annexe). Chaque segment de droite correspond à un système de production donné. Le graphique montre que les différents segments au sein de chacun des sous-ensembles herbagers et non herbagers s'ordonnent selon une pente décroissante à mesure que la gamme de superficie s'accroît, ce qui traduit le fait que les exploitations dotées d'une plus grande superficie par actif mettent en œuvre des systèmes qui se caractérisent par une intensité en travail et/ou en capital moindre par

Figure 2. Valeur ajoutée nette par actif en fonction de la superficie par actif des différents systèmes de production bovins laitiers identifiés

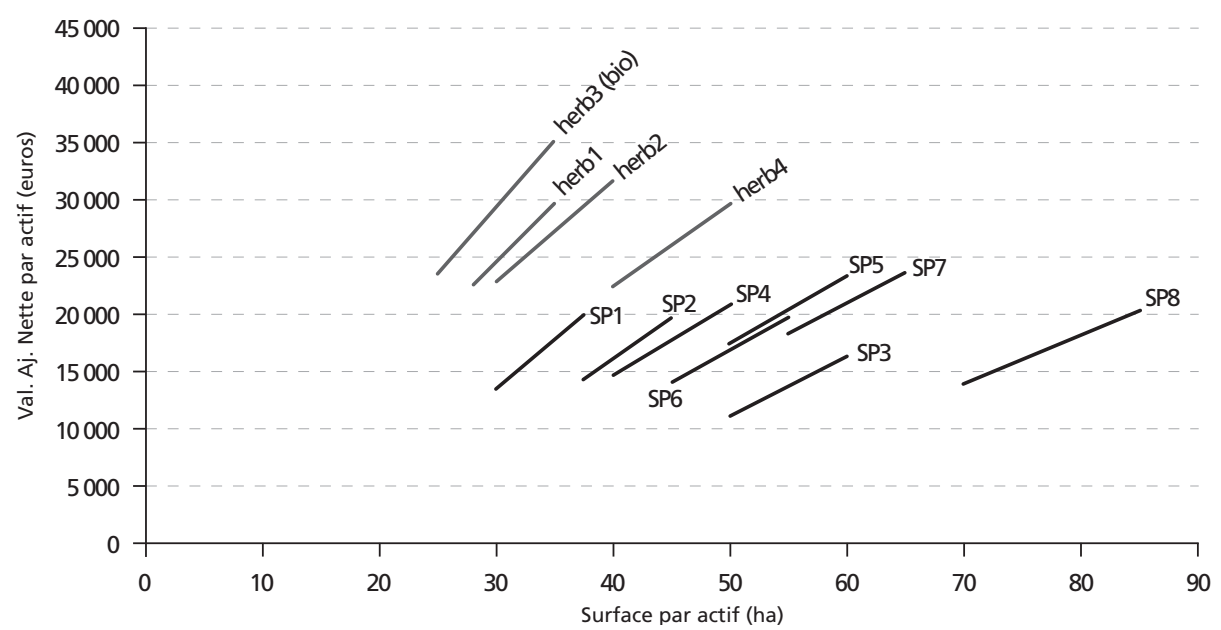

Source : calculs des auteurs 
Figure 3. Revenu brut par actif en fonction de la superficie par actif des différents systèmes de production bovins laitiers identifiés

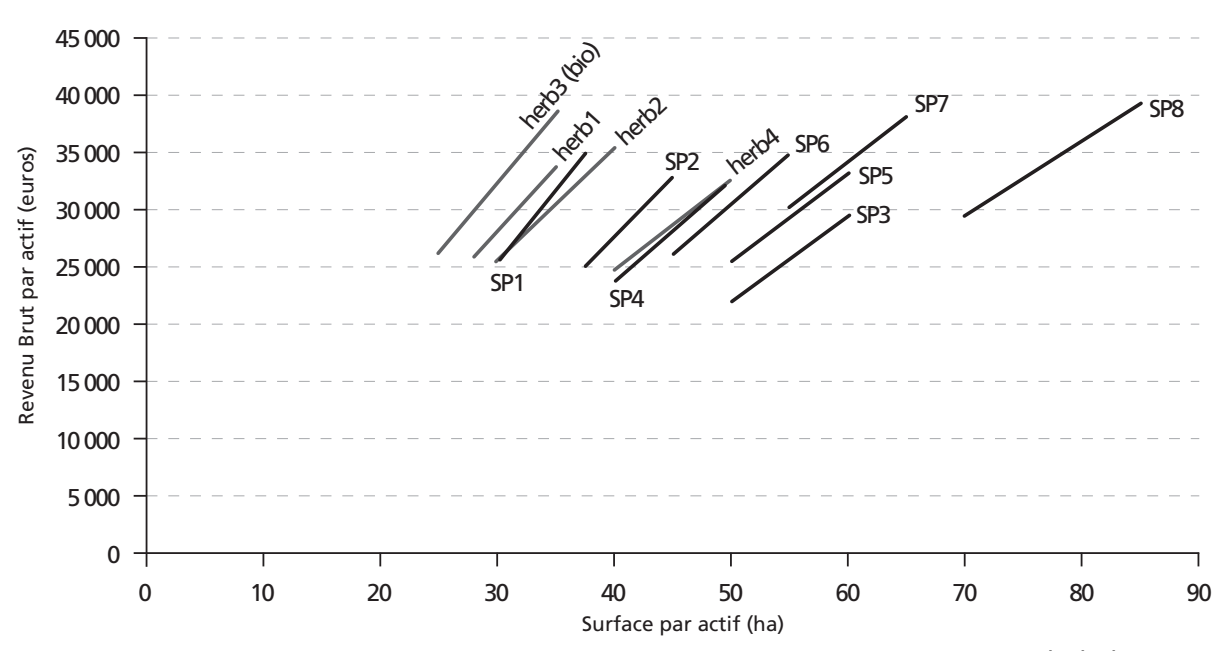

Source : calculs des auteurs

unité de surface et dégagent une valeur ajoutée nette et un revenu par hectare plus faibles (figure 2). Cependant les deux sousensembles se distinguent clairement par le fait que les systèmes herbagers à neuf mois de pâturage (herb1, herb2 et herb4, systèmes bénéficiant d'un prix du lait standard) permettent de dégager une valeur ajoutée nette par actif nettement supérieure à celle des autres systèmes de production (de 22000 à 32000 euros, contre 11000 à 24000 euros), sur des superficies par actif globalement moins importantes.

Malgré la valeur ajoutée nette par actif élevé qui les caractérise, les systèmes herbagers s'inscrivent dans une gamme de revenus bruts par actif semblable à celle des autres systèmes de la région (figure 3), situation qui s'explique par le fait qu'ils bénéficient d'un soutien par actif nettement moins élevé, de 6000 à 11000 euros, contre 17000 à 34000 euros pour les systèmes avec pâturage de printemps ou en zéro pâturage.

Des systèmes intensifs en termes de création de richesse

Les éleveurs herbagers ont ainsi réalisé une réduction de leurs coûts bien supé- rieure à celle de leur produit brut, ce qui leur permet de créer une valeur ajoutée par hectare élevée et in fine de disposer d'un revenu par hectare supérieur à celui des systèmes reposant peu sur le pâturage, en dépit d'un soutien bien moindre. Grâce à cette évolution positive, ils ont été en mesure de maintenir leur revenu tout en limitant leur agrandissement. La recherche d'un niveau relativement élevé de production réalisée de la façon la plus économe et autonome possible inscrit ces systèmes herbagers dans une démarche d'agroécologie, qui n'est "pas intéressée par la maximisation de la production d'une denrée particulière, mais plutôt par l'optimisation de l'ensemble de l'agro-écosystème » et où l' "accent est mis sur la pérennité écologique plutôt que sur la productivité à court terme »(Altieri, 1986).

Ces prairies d'association conduites sans azote de synthèse ont des effets précédents positifs pour la culture suivante dans la rotation sur l'état physique, chimique et biologique du sol : amélioration de la structure, et enrichissement en azote du sol, préservation de la micro- et macrofaune qu'il abrite (Voisin, 1960 ; Bourguignon, 
RECHERCHES

Nadège GARAMBOIS, Sophie DEVIENNE

Figure 4. Valeur ajoutée nette par litre de lait et production de lait par hectare de SAU et de SFP caractérisant les principaux types de systèmes de production

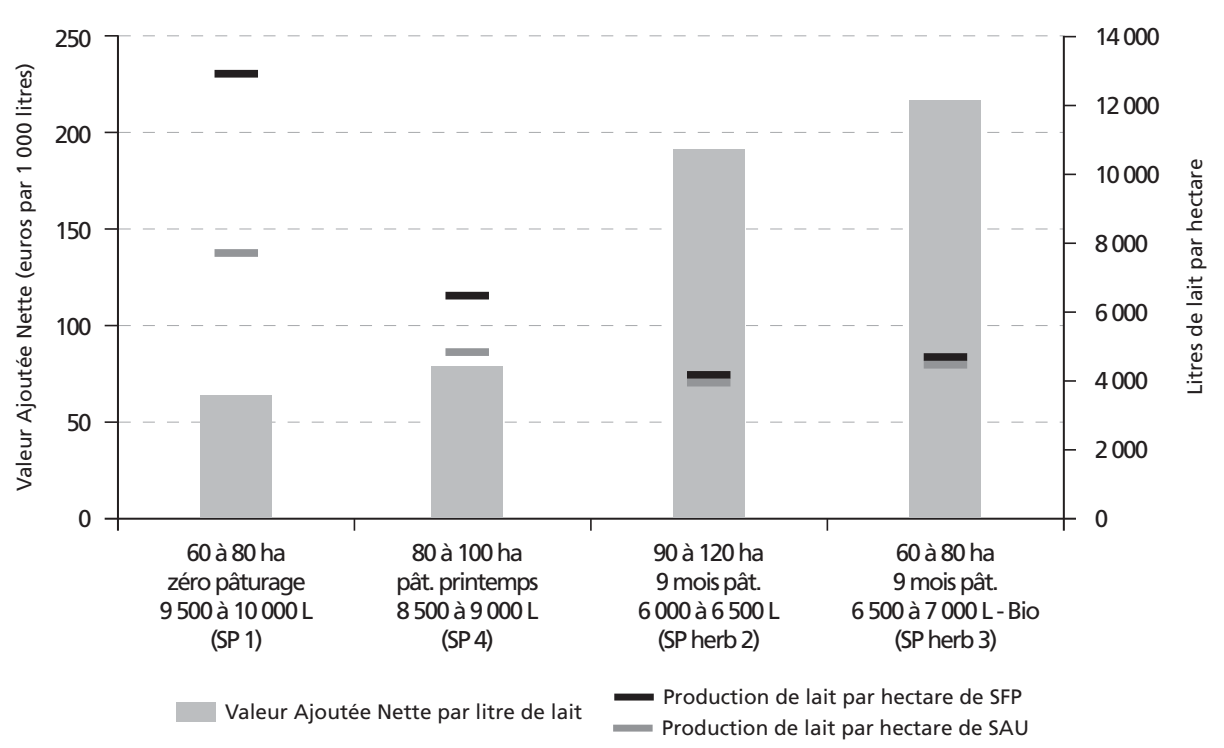

Source : calculs des auteurs

2002), lutte contre les adventices et élimination des pathogènes des cultures. Ces effets précédent ont permis aux éleveurs de réduire fortement les apports d'azote de synthèse ainsi que les applications de pesticides sur les cultures annuelles, tout en atteignant un rendement de 50 à 55 quintaux par ha (q/ha) pour les céréales à paille (au lieu de 65 à 70 q/ha dans la région) et en conservant une production moyenne de $12 \mathrm{tMS} /$ ha pour le maïs fourrage non irrigué. La VAN par litre de lait ${ }^{9}$ enregistrée en système herbager à neuf mois de pâturage bénéficiant d'un prix du lait standard (herb2) atteint deux à trois fois celle des systèmes reposant peu ou pas sur le pâturage (figure 4). Cet écart élevé ne s'explique pas seulement par la plus longue durée de pâturage. Il est aussi

9. La VAN par litre de lait isole ici la seule richesse créée par la production laitière et la vente des vaches de réforme. Elle ne tient pas compte des productions, consommations intermédiaires et consommations de capital fixes imputables aux activités d'engraissement (taurillons, génisses, bœufs) et aux grandes cultures. le résultat du caractère systémique des économies réalisées grâce au type de prairies implantées, à leur mode d'exploitation et aux rotations mises en œuvre. L'obtention d'un prix du lait supérieur grâce au passage en agriculture biologique (herb3) renforce cet écart, sans pour autant permettre une augmentation aussi ample.

Ces systèmes bovins laitiers herbagers, en permettant d'atteindre une valeur ajoutée par hectare et un revenu par hectare élevés, témoignent du fait que le recours à la prairie et au pâturage n'est pas nécessairement synonyme d'extensivité. Offrant des revenus par actif similaires à ceux des autres systèmes de production, pour des gammes de superficie équivalente ou inférieure malgré un niveau de subventions plus faible, ils montrent leur aptitude à maintenir des actifs moins soutenus financièrement sur de petites surfaces : il s'agit donc bien de systèmes intensifs, non pas sur le plan de la production, mais sur celui de la richesse créée par unité de surface. 
Figure 5. Évolution comparée par hectare et en monnaie constante du produit brut, des consommations intermédiaires et de capital fixe, de la valeur ajoutée nette et des subventions perçues* chaque année entre 1990 et 2009 , en système de production 6 et herbager 2 **
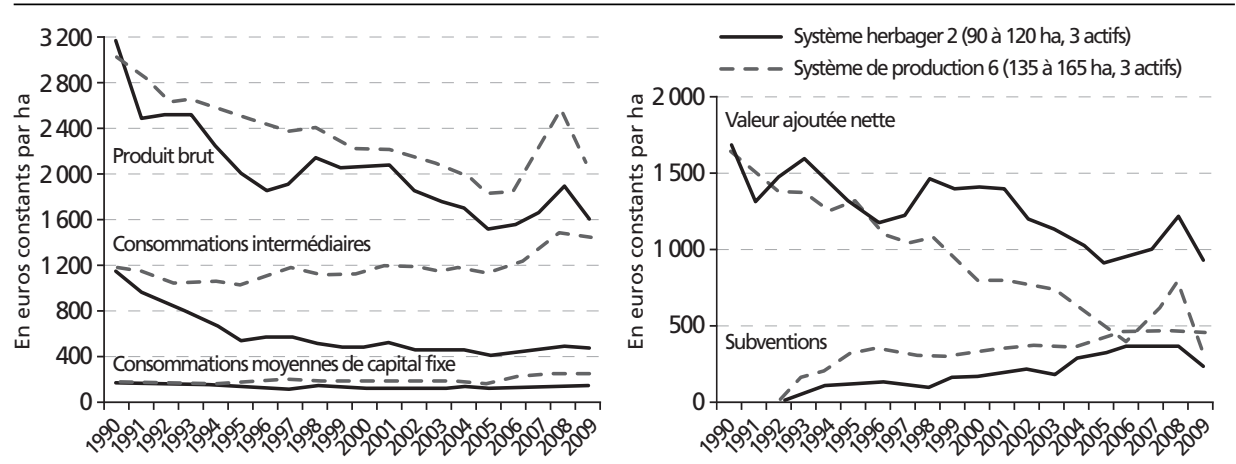

* CAD inclus en système herbager de 2004 à 2008 ; ** issus de la différenciation du même système de production initial à la fin des années 1980, mis en œuvre à l'époque au sein d'exploitations à trois actifs de 60 à 90 ha

Source : calculs des auteurs

\section{Les perspectives de l'élevage bovin laitier du Bocage vendéen Quelle place pour les pratiques herbagères économes?}

Le récent bilan de santé de la PAC, dont certains soutiens ont été réorientés vers l'« élevage à l'herbe » ne s'est pas traduit par un rééquilibrage en faveur de ces systèmes herbagers économes qui, du fait des surfaces moins importantes sur lesquelles ils sont mis en œuvre, continuent à subir de nettes inégalités de soutien. L'écart est particulièrement marqué avec le système de production à double spécialisation bovin laitier et grandes cultures au sein duquel l'actif agricole dégage en moyenne un produit brut double de celui atteint avec un système à neuf mois de pâturage, mais crée une valeur ajoutée $40 \%$ plus faible en mobilisant deux fois plus de surface agricole, pour dégager le même revenu par actif, mais comprenant trois fois plus de soutiens publics. Ces résultats locaux récents étayent ceux établis par Kroll, Mortaud et Thareau (1999) sur la base d'un vaste échantillon de données issues du Réseau d'information comptable agricole (Rica 1995) de l'Otex « lait » spécialisée, qui montraient la faible efficacité avec laquelle les vastes unités de production laitières peu autonomes valorisaient les aides publiques. Ils invitent également à reconsidérer l'assimilation souvent faite entre productivité physique et productivité économique du travail et à s'interroger sur la pertinence sociale, économique et écologique de cette logique de développement agricole.

La mise en œuvre dans le Bocage vendéen de ces systèmes herbagers économes, caractérisés par un ralentissement de la substitution de capital au travail, a conduit à freiner la diminution de la valeur ajoutée créée par hectare et par actif ces vingt dernières années (figure 5), permettant une progression moins rapide de la superficie par actif et ainsi, depuis 1990, le maintien en moyenne de $50 \%$ d'emplois agricoles en plus.

Grâce à un niveau d'investissement et à des besoins en trésorerie moindres, l'adoption de ces systèmes de production a par ailleurs constitué, pour certains agriculteurs initialement moins bien dotés en facteurs de production, une alternative viable à la cessation d'activité. Leur développement apparaît donc comme une option efficace pour limiter l'érosion du nombre d'actifs agricoles. Celle-ci nécessite cependant de bénéficier d'un parcellaire suffisamment groupé, ce qui, à l'exception de très vastes unités de production, est le cas 
de nombreuses exploitations dans cette région où le remembrement a été précoce et particulièrement abouti.

Ces systèmes herbagers économes permettent d'autre part de substantielles réductions au niveau de l'utilisation d'engrais azoté de synthèse, de carburant et de pesticides. Pour une même gamme de production laitière par hectare de SAU, les quantités d'ammonitrate et de pesticides consommées par litre de lait produit sont réduites d'un facteur dix et celle de carburant de $60 \%$. Par ailleurs, la très large majorité des exploitations en système herbager n'irrigue pas le maïs, dont la surface a de toute façon été massivement réduite. Enfin, le recours accru au pâturage implique le maintien et l'entretien des haies qui participent à la constitution d'un paysage bocager harmonieux (Béranger, 2002).

Les conditions d'installation en système herbager se révèlent favorables car le niveau de capital nécessaire à l'installation sur de plus petites structures, dont la logique de production nécessite peu de matériel, facilite les installations hors cadre familial. Le métier d'agriculteur proposé est d'autant plus attractif que le volume de travail est réduit (jusqu'à $25 \%$ de réduction de la durée hebdomadaire de travail dans le Bocage vendéen) et garantit une plus grande parité avec les autres secteurs d'emploi en termes de rémunération horaire. Les tâches sont différentes : le travail est plus régulier toute l'année et repose en grande partie sur une observation fine des prairies et l'acquisition de nouveaux savoir-faire permettant une conduite ajustée du troupeau au pâturage tournant sur prairies d'association. L'autonomie qu'il confère aux agriculteurs, tant dans le fonctionnement technique que dans la constitution du revenu, ainsi que la réduction de consommations d'intrants potentiellement polluants constituent autant d'éléments incitatifs pour des candidats à l'installation (Jouin, 1999).

Une diffusion récente d'une partie des pratiques économes développées s'observe néanmoins dans la région. Celle-ci consiste en l'introduction de trèfle blanc au sein des prairies temporaires de ray-grass anglais pur qui permet de réduire la fertilisation à base d'engrais azoté de synthèse. Ces initiatives demeurent marginales dans le fonctionnement des systèmes de production et insuffisantes pour conduire à un réel ralentissement de l'accroissement de la surface exploitée par actif et au maintien d'un plus grand nombre d'agriculteurs. Le découplage des aides PAC et la construction par les éleveurs d'un référentiel technique local contribuent néanmoins aujourd'hui à créer des conditions techniques et économiques qui semblent désormais plus incitatives au passage en système herbager. Celui-ci requiert néanmoins un savoir-faire particulier, qui demeure peu enseigné et vulgarisé dans les circuits classiques d'enseignement et de vulgarisation agricole (lycée agricoles, chambre d'agriculture, agrofourniture, contrôle laitier). Sans cet appui « institutionnel », beaucoup d'éleveurs n'osent vraisemblablement pas s'engager dans une refonte en profondeur de leur système.

\section{Conclusion}

L'analyse-diagnostic conduite dans la région a ainsi permis de mettre en évidence deux logiques de développement radicalement différentes : d'un côté des systèmes de production basés sur un niveau de production et de nombre d'animaux par hectare et par actif toujours plus élevés, grâce à un recours important aux consommations intermédiaires (engrais de synthèse, pesticides, compléments azotés, carburant...) et à l'adoption d'équipements performants et coûteux ; de l'autre des systèmes qui privilégient la recherche d'une haute valeur ajoutée par hectare par la diminution des consommations, grâce à l'utilisation de prairies associant graminées et légumineuses, et à une place très importante accordée au pâturage, dont la conduite s'appuie sur une 
observation attentive de la pousse de l'herbe afin d'optimiser l'utilisation des ressources de la prairie.

Les systèmes herbagers économes consistent à utiliser au mieux le fonctionnement de l'écosystème (cultures pluriannuelles, associations à base de légumineuses, utilisation de l'effet précédent cultural, récolte directe par les animaux...) en réduisant au maximum le recours à des intrants coûteux et dont, pour certains d'entre eux, les effets polluants ne sont plus à démontrer. Cette étude comparative a permis de montrer que, dans des conditions édaphiques qui ne sont pourtant pas optimales (déficit hydrique estival), ces systèmes herbagers relevant de l'agroécologie sont intensifs au plan de la création de valeur ajoutée et moins dépendants des aides publiques pour la réalisation de leur revenu. Leurs performances économiques élevées ont permis le maintien d'un plus grand nombre d'emplois agricoles et ne sont pas liées à des prix à la production supérieurs.

En l'absence, au début des années 1990, de référentiel technique local et d'un franc soutien institutionnel, leur mise au point doit beaucoup à la persévérance des éleveurs et à une réflexion de groupe. Dans un contexte de hausse du prix des matières premières et des dérivés du pétrole et à l'heure où l'agriculture doit plus que jamais prendre en compte les paramètres environnementaux, ces systèmes herbagers constituent une alternative innovante et performante, offrant aux éleveurs laitiers du Bocage vendéen un compromis rentable entre production et préservation des ressources. Si l'enjeu du développement agricole consiste désormais à utiliser au mieux le fonctionnement des écosystèmes sans en compromettre la reproduction (Griffon, 2010), ces systèmes invitent à réfléchir à l'adaptation de cette logique herbagère développée par certains éleveurs de l'Ouest, à la fois productive et économe, à d'autres régions d'élevage en France. Loin d'être un « paquet technique » à diffuser, les systèmes herbagers économes offrent une voie de réflexion stimulante pour la recherche. Encore faut-il pour promouvoir le développement de tels systèmes, qui conjuguent « productivité économique et pertinence écologique » (Hervieu, 2002), qu'une véritable recherche-développement soit mise en place afin d'aider à la définition de référentiels techniques adaptés aux conditions agropédologiques spécifiques de chaque région, accompagnée de mesures de politique agricole plus incitatives.

\section{RÉFÉRENCES BIBLIOGRAPHIQUES}

Alard V., Béranger C., Journet M. (2002). À la recherche d'une agriculture durable-Étude de systèmes herbagers économes en Bretagne. Paris, INRA Éditions, $340 \mathrm{p}$.

Altieri M.-A. (1986). L'agro-écologie. Bases scientifiques d'une agriculture alternative. Paris, Éditions Debard, 237 p.

Béranger C. (2002). La multifonctionnalité des prairies : acquis et interrogations $d u$ $19^{e}$ CEH. Paris, Fourrages, $\mathrm{n}^{\circ} 171, \mathrm{p}$. 227-237.
Boucarut J-M, Moyne V., Pollina L. (1996). L'agriculture depuis 1949 - Croissance des volumes, chute des prix. Paris, INSEE Première, $\mathrm{n}^{\circ}$ 430, $4 \mathrm{p}$.

Bourguignon C. (2002). Le sol, la terre et les champs. Paris, Ed. Sang de la terre, 190 p.

Cochet H., Devienne S. (2006). Fonctionnement et performances économiques des systèmes de production agricole : une démarche à l'échelle régionale. Cahiers Agriculture, vol. 15, n 6 , pp. 578-583. 
Cochet H., Devienne S., Dufumier M. (2007). L'agriculture comparée : une discipline de synthèse ?, Paris, Économie rurale, $n^{\circ}$ 297-298, pp. 99-112.

Civam (Centres d'Initiatives pour Valoriser l'Agriculture et le Milieu rural) (2009). Construire et conduire un système herbager économe. Cesson-Sévigné, Cahiers techniques de l'agriculture durable, RAD/FNCivam, $76 \mathrm{p}$.

Deléage E. (2004). Paysans, de la parcelle à la planète - Socio-anthropologie du Réseau d'agriculture durable. Paris, Syllepse, 245 p.

Desriers M. (2007). L'agriculture française depuis cinquante ans : des petites exploitations familiales aux droits à paiement unique. In « L'agriculture, nouveaux défis ». Paris, INSEE, coll. INSEE Références, pp 17-30.

Dufumier M. (1996). Les projets de développement agricole. Paris, CTA-Karthala, $354 \mathrm{p}$.

Dufumier M., Bergeret P. (2002). Analyser la diversité des exploitations agricoles. In Mémento de l'agronome. Paris, CIRAD - GRET - Ministère des Affaires étrangères, pp. 321-344.

Dumont R., Coléou J., Séverac G. (1957). Projet de rapport sur l'orientation et la modernisation agricole de la Loire-Atlantique et de la Vendée. Comité d'Expansion Économique Loire-AtlantiqueVendée, $97 \mathrm{p}$.

Garambois N. (2011). Des prairies et des hommes : agro-écologie, création de richesse et emploi en élevage bovin. Paris, Thèse de doctorat, AgroParisTech, 595 p.

Griffon M. (2010). Pour des agricultures écologiquement intensives. La Tour d'Aigues, Éditions de l'aube, 143 p.

Guihard V., Lesdos C. (2007). L'agriculture sur trente ans: une analyse comparative avec l'industrie et les services. In «L'agriculture, nouveaux défis ». Paris. INSEE, coll. INSEE - Références, pp. 47-63.
Hervieu B. (2002). Préface. In Agriculture, un tournant nécessaire, Groupe de Bruges. Paris, Éditions de l'Aube, 92 p.

Jouin C. (1999). De nouveaux paysans Une agriculture pour vivre mieux. Nantes, Siloë, 150 p.

Kroll J.-C., Mortaud J.-M., Thareau J. (1999). Performances économiques et aides publiques en production laitière. Paris, Économie rurale, $\mathrm{n}^{\circ} 253$, pp. 42-50.

Landais E. (1992). Principes de modélisation des systèmes d'élevage. Les cahiers de la Recherche-Développement, $\mathrm{n}^{\circ} 32$, pp. 82-95.

Le Rohellec C., Mouchet C. (2004). Évaluation de l'efficacité économique d'exploitations laitières en agriculture durable. Une comparaison aux références $d u$ RICA. Colloque SFER novembre 2004. $17 \mathrm{p}$.

Le Rohellec (2008). Efficacité économique des systèmes laitiers herbagers en agriculture durable (RAD) : une comparaison avec le RICA. Paris, Fourrages, $\mathrm{n}^{\circ} 193$, p. 107-113

Mazoyer M. (1982). Origines et mécanismes des inégalités régionales de développement agricole en Europe. Paris, Économie rurale, $\mathrm{n}^{\circ} 150-151$, pp. 25-33

Mazoyer M., Roudart L. (1997). Histoire des agricultures du monde, du néolithique à la crise contemporaine. Paris, Éditions du Seuil, 534 p.

Mounier A. (1992). Les théories économiques de la croissance agricole. Paris, Economica/INRA, 427 p.

Pochon A. (2002). La prairie temporaire à base de trèfle blanc, $3^{\mathrm{e}}$ édition mise à jour. Plérin, CEDAPA, 127 p.

Reboul C. (1976). Mode de production et systèmes de culture et d'élevage. Paris, Économie rurale, $\mathrm{n}^{\circ} 112$, pp. 55-65.

Voisin A. (1957). La productivité de l'herbe. Paris, Ed. La maison rustique, $467 \mathrm{p}$.

Voisin A. (1960). La dynamique des herbages. Paris, Ed. La maison rustique, $319 \mathrm{p}$. 


\section{ANNEXES}

\section{Détail des calculs du revenu brut par actif familial pour un système de production}

PB Produit Brut

$-\mathrm{CI}_{\mathrm{p}} \quad$ Consommations intermédiaires proportionnelles

$-\mathrm{CI}_{\mathrm{np}} \quad$ Consommations intermédiaires non proportionnelles

= VAB Valeur Ajoutée Brute

- $\mathrm{CK}_{\mathrm{p}}$ Consommations de capital fixe proportionnelles

- $\mathrm{CK}_{\mathrm{np}}$ Consommations de capital fixe non proportionnelles

= VAN Valeur Ajoutée Nette

- L Fermage (proportionnel à la surface)

- Int $\mathrm{p}_{\mathrm{p} / \mathrm{pp}}$ Intérêts des emprunts (proportionnels/non prop.)

- $\operatorname{Imp}_{\mathrm{p} / \mathrm{np}}$ Impôts et taxes (proportionnels/non prop.)

+ Subv Paiement de la main-d'œuvre salariée, charges sociales incluses (prop./non prop.) Subventions (généralement proportionnelles à la surface)

\section{$=\mathrm{RB}$ Revenu Brute agricole}

Pour chaque système de production, caractérisé par un niveau de ressources et une combinaison spécifique de systèmes de culture et d'élevage, la modélisation du fonctionnement technique du système de production ouvre la voie à une modélisation des résultats économiques en lien avec ce fonctionnement technique. Il est possible à partir de la conduite précise des différents systèmes de culture et d'élevage d'écrire les principales constantes économiques caractéristiques de ce système de production par hectare ou par vache laitière.

Exemple de calcul pour une culture d'un système de culture pratiqué dans un système de production donné :

$\mathbf{P B} / \mathbf{h a}=$ rendement à l'hectare (qui dépend de la localisation des parcelles, des itinéraires techniques et de la succession culturale pratiqués) $\times$ prix moyen (qui dépend de la période de vente, de la qualité du produit, etc.)

$\mathbf{C I} / \mathrm{ha}=$ frais de semences, engrais, amendements, pesticides, CUMA et entreprise par hectare (qui dépendent des itinéraires techniques spécifiques à ce système de culture et à ce système de production (selon les équipements en propriété dont disposent les agriculteurs), tant pour les quantités consommées que pour la nature des biens et leur prix unitaire)

Un raisonnement semblable permet de calculer ces grandeurs par vache pour chaque système d'élevage, sur la base de ses caractéristiques techniques.

Les équipements et bâtiments sont une caractéristique de chaque système de production et leur dépréciation annuelle liée à l'usure (qui équivaut à une consommation annuelle moyenne de capital fixe) est facilement évaluable :

$\mathrm{CK}_{\mathrm{p}} / \mathrm{ha}=$ (prix d'achat - prix de revente) / nombre d'année d'usage, exprimé en monnaie constante et par hectare

Sur la base des principales constantes économiques calculées par hectare ou par vache laitière, il est possible pour un système de production donné de représenter la Valeur Ajoutée Nette par actif et le Revenu Brut agricole par actif familial en fonction de la superficie/actif de manière simple :

$$
\begin{aligned}
& \text { VAN/A }=\left(P B / h a-C I_{p} / h a-C K_{p} / h a\right) \times S / A-\left(C I_{n p}+C K_{n p}\right) / A \\
& R B / A_{f}=\left(P B / h a-C I_{p} / h a-C K_{p} / h a-L / h a-I n t_{p} / h a-I m p_{p} / h a-M w_{p} / h a+S u b v\right) \times S / A_{f}- \\
& \left(\mathrm{CI}_{\mathrm{np}}+\mathrm{CK}_{\mathrm{np}}-\mathrm{Int}_{\mathrm{np}} / \mathrm{ha}-\mathrm{Imp}_{\mathrm{np}} / \mathrm{ha}-\mathrm{Mw}_{\mathrm{np}} / \mathrm{ha}\right) / \mathrm{A}
\end{aligned}
$$

où : $\quad$ A est le nombre d'actifs de l'exploitation et $\mathrm{A}_{\mathrm{f}}$ celui des seuls actifs familiaux ;

$\mathrm{S} / \mathrm{A}$ et $\mathrm{S} / \mathrm{A}_{\mathrm{f}}$ sont respectivement les superficies par actif et par actif familial. 